\title{
Constitutive Expression of OsGH3.1 Reduces Auxin Content and Enhances Defense Response and Resistance to a Fungal Pathogen in Rice
}

\author{
Concha Domingo,, ${ }^{1}$ Fernando Andrés, ${ }^{1}$ Didier Tharreau, ${ }^{2}$ Domingo J. Iglesias, ${ }^{1}$ and Manuel Talón ${ }^{1}$ \\ ${ }^{1}$ Instituto Valenciano de Investigaciones Agrarias. Carretera Moncada-Naquera Km 7.5, 46113 Moncada, Spain; ${ }^{2} \mathrm{CIRAD}$, \\ UMR BGPI, CIRAD-INRA-SupAgro.M, TA A-54/K, 34398 Montpellier, France
}

Submitted 6 August 2008. Accepted 5 October 2008.

GH3 genes are main components of the hormonal mechanism regulating growth and development and, hence, are deeply involved in a broad range of physiological processes. They are implicated in hormonal homeostasis through the conjugation to amino acids of the free form of essential plant growth regulators such as indoleacetic and jasmonic acids. In this work, we showed that $O s G H 3.1$ overexpression in rice caused dwarfism and significantly reduced both free auxin content and cell elongation. Functional classification of the transcriptomic profiling revealed that most genes involved in auxin biosynthesis and auxin signaling inhibition were induced and repressed, respectively. Many genes related to cell organization and biogenesis were also significantly downregulated. The survey also showed that, although the response to abiotic stresses was not clearly stimulated, $O s G H 3.1$ overexpression did activate a significant number of defense-related genes. In successive bioassays, it was demonstrated that the resistance of rice plants to pathogen infection, evaluated with two different Magnaporthe grisea strains, was higher in the transformants overexpressing $O_{s} G H 3.1$. Taken together, these results indicate that $O s G H 3.1$ overexpression reduces auxin content, inhibits cell growth and cell wall loosening, and enhances resistance to a fungal pathogen. Our results provide evidence that auxin homeostasis can regulate the activation of the defense response in rice.

Additional keywords: fungal resistance.

It is well established that hormones regulate many aspects of the natural programs for plant growth and development (Leyser 2006; Teale et al. 2006). Many studies support the idea that plant signaling pathways are composed of intricate networks with frequent and highly coordinated interplays allowing coordinate and appropriate responses to the different types of environmental stimuli (Achard et al. 2006; Fu and Harberd 2003; Halliday 2004). Thus, there is wide evidence that hormones regulate plant responses to the environment, especially those responses related to life cycles and adverse conditions for growth, including many stresses. Evidence for the involvement of jasmonic acid (JA), ethylene, and salicylic acid (SA) in bi-

Corresponding author: C. Domingo; Telephone: +34 963424 000; E-mail: Domingo_concar@gva.es

* The $\boldsymbol{e}$-Xtra logo stands for "electronic extra" and indicates that two supplemental figures and five supplemental tables are published online. otic stress is ubiquitous (Anderson et al. 2004; McConn et al. 1997; Ryals et al. 1996). In recent years, however, novel observations have been presented indicating that other hormonal compounds, especially auxin, are apparently implicated in the plant responses to stress. Thus, it was suggested that auxin homeostasis was one of the components participating in the regulation of the overall defense response during viral systemic infections (Mayda et al. 2000). It has also been shown that antibacterial resistance is enhanced by repressing auxin signaling in Arabidopsis (Navarro et al. 2006). Furthermore, there is evidence that the presence of bacterial effector molecules modulates host auxin physiology to promote disease (Chen et al. 2007). It is also known that nontumorigenic Pseudomonas syringae and Bacillus strains can produce auxins (Glickmann et al. 1998; Idris et al. 2007) or even gibberellins (GutierrezMañero et al. 2001) at high levels, suggesting that plant pathogens can manipulate their host resistance through hormonal production or regulation. It has also been demonstrated that the inhibition of auxin responses is a part of the SA-mediated disease-resistance mechanism in Arabidopsis (Wang et al. 2007). $\mathrm{SA}$ is a signal molecule for systemic acquired resistance in dicots but its role is still uncertain in rice because it maintains a high endogenous level of SA without activating defense response (Silverman et al. 1995). Collectively, these observations indicate that the reduction of auxin effects triggers several defense responses, particularly against viral and bacterial pathogens. To date, however, the response to fungal resistance to auxin depletion is not known. To this end, a strategy involving transgenic $G H 3$ rice was developed.

The GH3 family consists of a set of genes activated rapidly in the primary response of plants to auxin. The $G H 3$ gene family in Arabidopsis is implicated in hormonal homeostasis through conjugation to amino acids of the free form of indoleacetic acids, SA, and JA (Staswick et al. 2005). In Arabidopsis, the GH3 gene family is composed of 19 members. In rice, 13 putative members of this family were identified based on sequence similarity. These displayed open reading frames (ORF) of similar size and variable exon organization (Jain et al. 2006a; Terol et al. 2006). Expressed sequence tag analysis and reverse-transcriptase polymerase chain reactin (RT-PCR) assays indicated that 12 of the hypothetical ORF were active genes, and phylogenetic clustering showed a high degree of conservation between the rice and Arabidopsis GH3 proteins. Rice proteins, however, were present in only two of the three Arabidopsis groups (i.e., groups I and II). AtGH3.11 (JAR1/FIN219) is a member of group I and showed JA-amino synthetase activity (Staswick and Tiryaki 2004). Recently, it has been demonstrated that AtGH3.12, included in group III, 
regulates the SA-dependent defense response controlling pathogen-inducible SA levels (Jagadeeswaran et al. 2007; Nobuta et al. 2007). Many members of group II, including AtGH3.6 (DFL1), AtGH3.5 (AtGH3a), and AtGH3.2 (YDK1), were able to adenylate indole-3-acetic acid (IAA) and to catalyze IAA conjugation to amino acids through amide bounds (Staswick et al. 2005). Thus, YDK1 in Arabidopsis has been described as a negative regulator of growth and its overexpression produces a dwarf phenotype in Arabidopsis adult plants (Takase et al. 2004). DFL1 appears also to be a negative regulator of hypocotyl elongation (Nakazawa et al. 2001). The $d f l 1$ mutants overexpressing DFLl showed short hypocotyls under light conditions but not when grown in the dark. Its overexpression caused an exaggerated dwarf phenotype in the adult plant by inhibition of cell elongation in shoots. Surprisingly, free auxin levels were not significantly decreased in DFL1overexpressing plants although, in contrast, the level of IAAAsp conjugate was increased three- to fourfold compared with control plants (Staswick et al. 2005). AtGH3.5 adenylated both IAA and SA in vitro (Staswick et al. 2005) and it has been demonstrated that AtGH3.5 acts as a bifunctional modulator in both SA and auxin signaling during pathogen infection (Zhang et al. 2007). Few reports have been published related to the role of the GH3 family in rice. Recently, it has been shown that OsGH3.8 prevents free IAA accumulation and activates disease resistance to a bacterial pathogen through SA and JA signaling-independent pathways. Overexpression of $O s G H 3.8$ resulted in the suppression of auxin signaling, and as a consequence, abnormal plant morphology and also enhanced resistance to the rice pathogen Xanthomonas oryzae. It was suggested that these abnormalities in OsGH3.8-overexpressing plants were produced by the inhibition of expansin expression that prevents cell wall loosening (Ding et al. 2008).

In the work described here, OsGH3.1, the counterpart of the Arabidopsis DFL1, identified among the rice $O s G H 3$ genes by phylogenetic proximity (Terol et al. 2006), has been overexpressed in rice. Transgenic plants were then generated with a double objective: to i) confirm the anticipation that $O s G H 3.1$ overexpression reduces endogenous auxin levels and ii) elucidate whether or not auxin depletion enhances defense responses and, in particular, tolerance to fungal pathogens.

\section{RESULTS}

OsGH3.1 overexpression in rice causes a dwarf phenotype and depletion in free auxin.

We generated transgenic rice plants by introducing OsGH3.1 (Os01g57610) under the regulation of the Cauliflower mosaic virus $35 \mathrm{~S}$ promoter in rice cv. Bomba and examined 17 independent T1 lines. Transformants were classified into two different groups according to the severity of the dwarfism (Fig. 1). Approximately half of these plants showed a bushy severe dwarf phenotype, were shorter than $48 \mathrm{~cm}$ (control was 145 $\mathrm{cm}$ high), showed flowering delay of several months, and, in some cases, developed either sterile flowers or flowers with very few seed or multiple secondary tillers. The other half of the transgenic plants showed a mild phenotype, were shorter than control plants (falling into the range of 70 to $100 \mathrm{~cm}$ ), did not show any morphological abnormality and had normal flowering. Transgenic plants showed different levels of OsGH3.1 expression and the highest levels corresponded to shorter plants (Fig. 1B). Similar results were obtained with transgenic plants of cv. Bahia (data not shown).

Gas chromatography (GC) single-ion-monitoring mass spectrometry (MS) determinations showed that free IAA levels were significantly lower in T1 35S:OsGH3.1 plants (Table 1). Severe dwarf lines (for example, lines 18 and 36 that were 24 and $34 \mathrm{~cm}$ tall, respectively) had lower IAA levels than mild lines such as line 8 or Bomba controls that were taller (95 and $147 \mathrm{~cm}$, respectively). Lines showing severe dwarfism did not produce enough seed for further analysis; therefore, confirmation of reduction of the free IAA level in transgenic lines was obtained by analyzing additional T2 lines showing mild phenotypes. IAA content of 4-week-old Bomba and T2 lines is

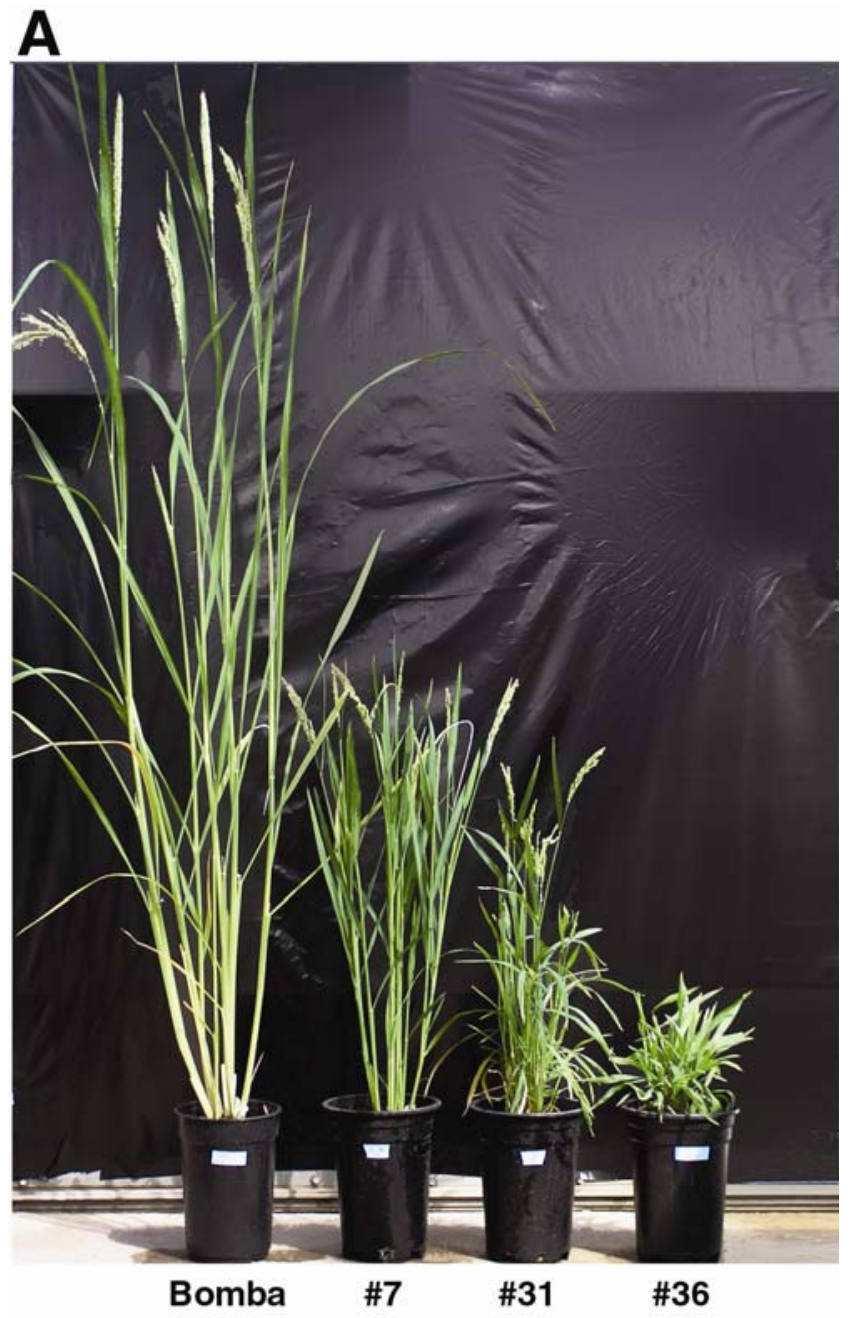

B

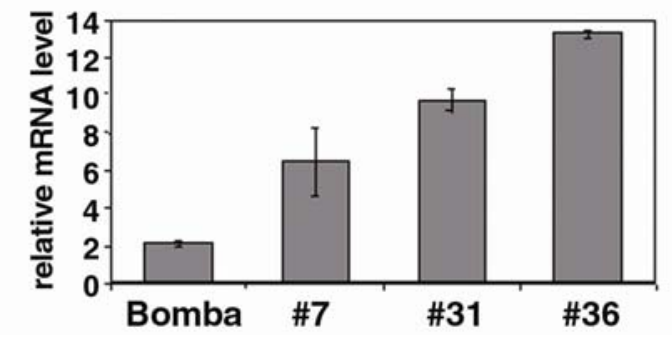

Fig. 1. Mature transgenic rice plants overexpressing OsGH3.1. A, Phenotype of untransformed control plants and transgenic lines 7, 31, and 36 (from left to right). Transformants were classified into two different groups: mild (lines 7 and 31) and severe (line 36) dwarfs. Mild dwarfs did not show any morphological or flowering abnormality whereas severe dwarfs showed flowering delay and developed sterile flowers and multiple secondary tillers. B, OsGH3.1 expression in Bomba control plants and transformed lines 7, 31, and 36. Adults T1 lines were grown under greenhouse conditions and $O s G H 3.1$ expression was analyzed by quantitative reversetranscriptase polymerase chain reaction using primers amplifying fragments from the coding sequence region. 
shown in Table 1. Young plants showed a lower IAA level than adult plants. It could be observed that plant height in Bomba and T2 lines 8, 13, and 31 certainly correlated positively and negatively with IAA content and OsGH3.1 mRNA levels, respectively.

To know whether cell elongation was affected, sheaths from T2 adult plants exhibiting a mild dwarf phenotype (line 31) and from Bomba control were studied under an optical microscope. Cells in transgenic plants $(56.3 \pm 7.4 \mu \mathrm{m})$ were $46.0 \%$ shorter than those in Bomba untransformed plants (122.3 \pm $23.1 \mu \mathrm{m})$ (Fig. 2).

Arabidopsis dominant mutant dfl1-D showed a short-hypocotyl phenotype in light but not in darkness, indicating that AtGH3.6 inhibits cell elongation depending on light factors (Nakazawa et al 2001). To ascertain the response to light of rice plants overexpressing the rice $O s G H 3.1$, T2 seed were grown under either continuous light or dark conditions for 10 days and the aerial part was then measured. Under these conditions, no difference in length was observed with control seedlings (data not shown). However, when plants were grown to maturity under standard growth conditions, the final height of 35S:OsGH3.1 plants was shorter than control untransformed plants.

\section{Gene expression profile.}

Transgenic 35S:OsGH3.1 rice plants (line 31) were grown under standard growth conditions for 6 weeks and gene expression profiling was then analyzed. At this stage, differences in size between transgenic and wild-type plants were already detectable. For the microarray analysis, six independent hybridizations were performed using three different biological replicas following a dye-swap loop design. Data were normalized with the LIMMA package (Smyth 2005). RNA was isolated from transgenic or wild-type plants, amplified, and labeled with either $\mathrm{Cy}-3$ or $\mathrm{Cy}-5$. Labeled samples were hybridized to the $20 \mathrm{~K}$ NSF microarrays. In total, 700 genes on the rice cDNA microarray showed differential expression of twofold $(-1>M>$ 1 and $P$ value $<0.001$ ); 321 and 379 genes were up- and downregulated, respectively. Functional classification of genes was performed using the Plant GOSlim Assignment of Rice Proteins from TIGR. Of the 700 genes that showed significant variation, $236(33.7 \%)$ showed no Gene Ontology (GO) assignation and, from the remaining 464 genes, 219 were upregulated and 245 were downregulated. Microarray data was validated with quantitative (Q)-RT-PCR analyses (Supplementary Fig. S1).

Table 1. Plant height, indole-3-acetic acid (IAA) contents and relative mRNA from 35S:OsGH3.1 transgenic plants and from control (untransformed Bomba) $)^{2}$

\begin{tabular}{|c|c|c|c|}
\hline Plants & $\begin{array}{l}\text { Plant height } \\
\text { (cm) }\end{array}$ & $\begin{array}{l}\text { IAA contents } \\
\text { (ng } \text { g }^{-1} \text { fresh } \\
\text { weight) }\end{array}$ & $\begin{array}{c}\text { Relative } \\
\text { OsGH3.1 } \\
\text { (mRNA level) }\end{array}$ \\
\hline \multicolumn{4}{|l|}{ Adult } \\
\hline Bomba & 147.0 & $592.8 \pm 93.4 \mathrm{a}$ & $\ldots$ \\
\hline Line 8 (T1) & 95.0 & $404.4 \pm 46.5 b$ & $\ldots$ \\
\hline Line $18(\mathrm{~T} 1)$ & 24.5 & $280.6 \pm 63.8 \mathrm{c}$ & $\ldots$ \\
\hline Line 36 (T1) & 34.0 & $233.2 \pm 41.2 \mathrm{c}$ & $\ldots$ \\
\hline \multicolumn{4}{|l|}{ Four weeks old } \\
\hline Bomba & $45.6 \pm 1.9 \mathrm{a}$ & $320.3 \pm 22.8 \mathrm{a}$ & $31.7 \pm 3.5 \mathrm{c}$ \\
\hline Line 8 (T2) & $38.0 \pm 1.0 \mathrm{~b}$ & $254.3 \pm 25.4 \mathrm{ab}$ & $67.6 \pm 16.4 b$ \\
\hline Line $13(\mathrm{~T} 2)$ & $37.1 \pm 1.2 \mathrm{~b}$ & $211.2 \pm 17.9 \mathrm{~b}$ & $58.9 \pm 0.9 \mathrm{~b}$ \\
\hline Line 31 (T2) & $34.8 \pm 0.9 b$ & $234.5 \pm 25.2 b$ & $104.8 \pm 9.3 \mathrm{a}$ \\
\hline
\end{tabular}

Biological process classification according to their GO annotation of the gene set differentially expressed revealed few obvious categories. Genes most likely involved in i) auxin metabolism and perception, ii) growth and cellular morphogenesis, and iii) stress related were studied in more detail.

\section{Auxin metabolism and perception genes.}

Because 35S:OsGH3.1 plants showed lower free IAA levels, it was of interest to study the differential expression of genes related to the homeostasis, perception, and response to auxin in the microarray analysis. Microarray analysis showed that three auxin biosynthesis-related genes (Table 2) as well as seven other genes implicated in the biosynthesis of tryptophan, the precursor of IAA, were clearly induced (Table 3; Supplementary Table S1).

The survey revealed that at least six genes of the auxin-mediated signaling pathway were clearly downregulated in transgenic plants. Differential expression of some of these genes obtained by microarray analyses was further validated by QRT-PCR (Fig. 3). Four of these genes, OsIAA13, OsIAA17, OsIAA24, and OSIAA30, belong to the AUX/IAA family, a group of early auxin-responsive genes that are involved in the inhibition of auxin response (Hagen and Guilfoyle 2002; Jain et al. 2006b). Interestingly, the expression level of other Aux/IAA genes, such as OsIAA1, was not affected in transgenic plants (Fig. 3; Supplementary Table S4). Other downregulated gene were involved in auxin signaling, such as a putative homologue of ARF16 (Table 2). It has been shown that the expression of some $O s G H 3$ genes is regulated by $A R F 8$ (Tian et al. 2004). Because $A R F 8$ was not represented in the microarray, we also determined its expression level by Q-RT-PCR. The data presented in Figure 3 show that $A R F 8$ expression was reduced (threefold) in the transgenic plants. Microarray data showed no significant differential expression level $(\mathrm{M}=0.84)$ of transport inhibitor response 1 (TIRl), an F-box protein mediating Aux/IAA protein degradation (Gray et al. 2001), al-
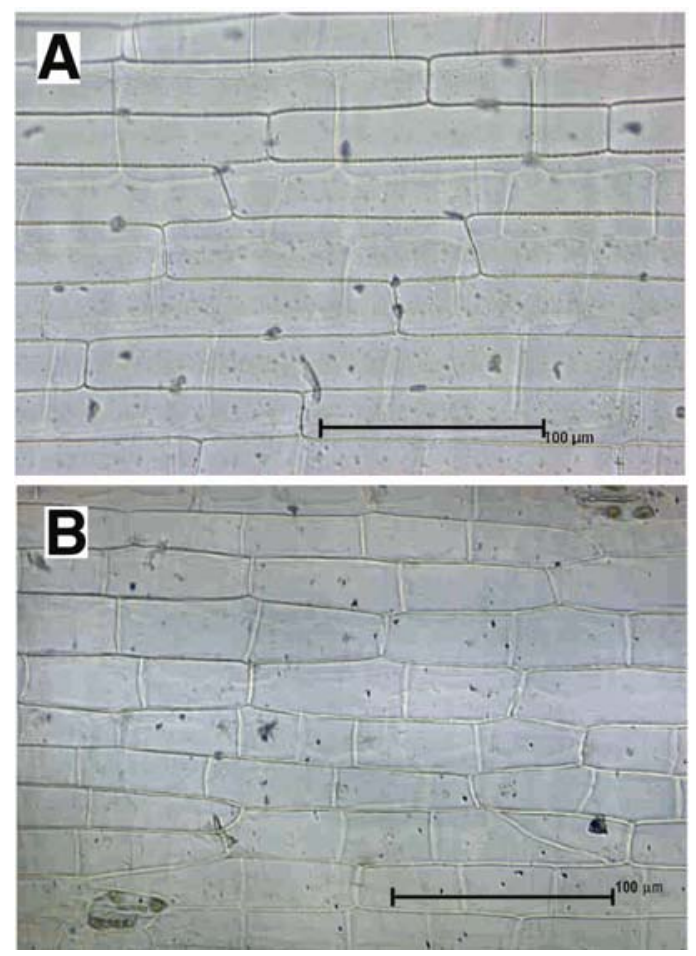

Fig. 2. Epidermal cells in A, control untransformed plants and $\mathbf{B}, 35 \mathrm{~S}$ : OsGH3.1 plants line 31. Sheath segments from second leaves of adult plants were studied with a Leica microscope. 
though Q-RT-PCR analysis revealed a slight decrease (1.5fold) (Fig. 3).

According to the microarray analyses, levels of other $O s G H 3$ transcripts were unchanged. To ascertain alteration of endogenous $O s G H 3.1$ transcript levels, distinguishing between both endogenous and transgene transcripts, Q-RT-PCR was performed with pairs of specific primers amplifying the untranslated region, which was not included in the 35S:OsGH3.1 sequence. Data in Figure 3 show no changes in the endogenous $O s G H 3.1$ transcripts in the transgenic plants.

Overall, the data obtained with these analyses indicated that auxin biosynthesis-related genes and mostly Aux/IAA genes, implicated in the inhibition of the auxin-signaling pathway, were induced and repressed, respectively, in OsGH3.1 transformants.

\section{Growth and cellular morphogenesis and metabolism related genes.}

The microarray analyses also revealed that there was a large number of differentially expressed genes related to growth and cellular morphogenesis. Most of them were downregulated. According to the GO functional classification, the highest proportion of differential expressed genes were associated with the "cellular organization and biogenesis" category (Table 4). Among them, several genes belonging to the cell growth (five upregulated versus 15 downregulated) and extracellular matrix organization and biogenesis (two upregulated versus 14 down- regulated) categories were found. These categories grouped cell wall loosening genes (mostly coding for expansins, cell wall biosynthesis genes, glycosyl transferases and hydrolases; and proline-rich proteins). The group of genes related to the cytoskeleton organization was also downregulated and included genes coding for tubulins and actin and genes involved in regulation of actin filament polymerization. Other groups of downregulated genes coded for protein-binding domains such as GTP-binding domain.

Upregulated genes in the growth and cellular morphogenesis functional category had mostly unrelated functions and could not be grouped in this way. Only a small set of three genes coding for cell-wall-associated proteins could be clustered together; these proteins are part of kinase complexes. Taken together, the microarray survey suggested that, in general, the transcripts associated with cellular growth were mostly downregulated in the transgenic plants.

A group of genes roughly related to the metabolism of nitrogen was also induced (Table 3). Within this group, seven genes were implicated in tryptophan biosynthesis, the precursor of IAA.

\section{Stress-related genes.}

A large cluster of differentially regulated genes involved in stress response was upregulated in 35S:OsGH3.1 plants (Table 4). It is remarkable that three out of the five genes most differentially upregulated coded for proteins related to defense re-

Table 2. Genes related to auxin biosynthesis and response differentially expressed in $O s G H 3.1$-overexpressing plants based on microarray analyses

\begin{tabular}{|c|c|c|c|c|}
\hline Locus & Putative function & $\mathbf{M}^{\mathbf{z}}$ & $P$ value & $\begin{array}{l}\text { Adjusted } \\
P \text { value }\end{array}$ \\
\hline \multicolumn{5}{|l|}{ Auxin biosynthesis } \\
\hline LOC_Os02g20360 & Tyrosine/nicotianamine aminotransferases family protein, expressed & 2.563 & $6.922 \mathrm{E}-36$ & $1.140 \mathrm{E}-33$ \\
\hline LOC_Os08g23150 & $\begin{array}{l}\text { Indole-3-glycerol phosphate synthase, chloroplast precursor, } \\
\text { putative, expressed }\end{array}$ & 1.299 & $2.321 \mathrm{E}-10$ & $5.705 \mathrm{E}-09$ \\
\hline LOC_Os07g18120 & Aldehyde oxidase 3 , putative, expressed & 0.994 & $1.239 \mathrm{E}-06$ & $1.579 \mathrm{E}-05$ \\
\hline LOC_Os02g19970 & Tyrosine/nicotianamine aminotransferases family protein, expressed & -1.063 & $1.859 \mathrm{E}-05$ & $1.861 \mathrm{E}-04$ \\
\hline \multicolumn{5}{|l|}{ Auxin transport } \\
\hline LOC_Os06g08080 & $\begin{array}{l}\text { Pyrophosphate-energized vacuolar membrane proton pump, } \\
\text { putative, expressed }\end{array}$ & -1.600 & $5.761 \mathrm{E}-15$ & $2.532 \mathrm{E}-13$ \\
\hline \multicolumn{5}{|c|}{ Auxin mediated signaling pathway } \\
\hline LOC_Os07g08460 & OsIAA24 & -0.933 & 5.290E-06 & $5.931 \mathrm{E}-05$ \\
\hline LOC_Os05g14180 & OsIAA17 & -0.981 & 7.815E-05 & $6.770 \mathrm{E}-04$ \\
\hline LOC_Os02g02840 & RAC-like GTP binding protein RHO1, putative, expressed & -0.985 & $1.547 \mathrm{E}-06$ & $1.942 \mathrm{E}-05$ \\
\hline LOC_Os04g43910 & Auxin response factor 16 , putative, expressed & -1.031 & 4.909E-07 & $6.774 \mathrm{E}-06$ \\
\hline LOC_Os03g53150 & OsIAA 13 & -1.387 & $1.301 \mathrm{E}-11$ & $3.739 \mathrm{E}-10$ \\
\hline LOC_Os12g40890 & OsIAA30 & -1.492 & 3.324E-13 & $1.201 \mathrm{E}-11$ \\
\hline
\end{tabular}

${ }^{\mathrm{z}}$ Logarithm of fold change.

Table 3. Distribution of significant genes related to growth and cellular morphogenesis and metabolism, based on microarray analyses in transgenic plants overexpressing $O s G H 3.1^{2}$

\begin{tabular}{|c|c|c|c|}
\hline Functional category & Upregulated & Downregulated & Total \\
\hline \multicolumn{4}{|l|}{ Cell organization and biogenesis } \\
\hline Organelle organization and biogenesis & 4 & 14 & 18 \\
\hline Cell growth & 5 & 15 & 20 \\
\hline External encapsulating structure organization and biogenesis & 2 & 14 & 16 \\
\hline Cytoskeleton organization and biogenesis & 1 & 7 & 8 \\
\hline Morphogenesis & 6 & 23 & 29 \\
\hline Cell differentiation & 0 & 5 & 5 \\
\hline \multicolumn{4}{|l|}{ Metabolism } \\
\hline Cellular metabolism & 95 & 82 & 178 \\
\hline Primary metabolism & 79 & 71 & 150 \\
\hline Nitrogen compound metabolism & 12 & 1 & 13 \\
\hline Tryptophan biosynthesis & 7 & 0 & 7 \\
\hline Secondary metabolism (phenylpropanoids and terpenoids) & 20 & 9 & 29 \\
\hline Macromolecule metabolism & 31 & 43 & 74 \\
\hline Regulation of metabolism & 16 & 14 & 30 \\
\hline Catabolism & 6 & 6 & 12 \\
\hline
\end{tabular}

\footnotetext{
${ }^{\mathrm{z}}$ Genes were grouped in functional categories according to the Gene Ontology classification.
} 
sponse. In general, the responses of genes known to be involved in responses to biotic stress were clearly enhanced in the transformants. Thus, in all functional categories included in the biotic stress, the quantity of induced genes was higher than the amount of repressed genes. The number of upregulated genes related to pest, pathogen, or parasite, for example, was 2.6-fold higher than the number of genes downregulated (26 up versus 10 down) (Table 4). The induced group included several genes coding for different pathogenesis-related (PR) proteins such as PR-5, P21, three PR-10/BetvI family genes, two endochitinases, and three glucan-endo-1,3- $\beta$-glucosidases as well as one lipoxygenase and three peroxidases. Furthermore, six induced genes were associated with terpenoids metabolism, compounds that are related to plant stress responses (Table 3). Therefore, the microarray analyses indicated that transcripts associated with the responses to biotic stresses were clearly induced in OsGH3.1 overexpressing transgenic plants.

On the other hand, several genes known to be involved in response to abiotic stresses were differentially expressed in transgenic plants. The distribution in functional categories, according to GO classification, showed small subgroups with a different tendency in the number of induced or repressed genes (Table 4). Gene repression for cold response and gene induction for wounding response, for example, were particularly prominent whereas, in other groups of genes, such as those related to salt stress or water deprivation response, the variation in the
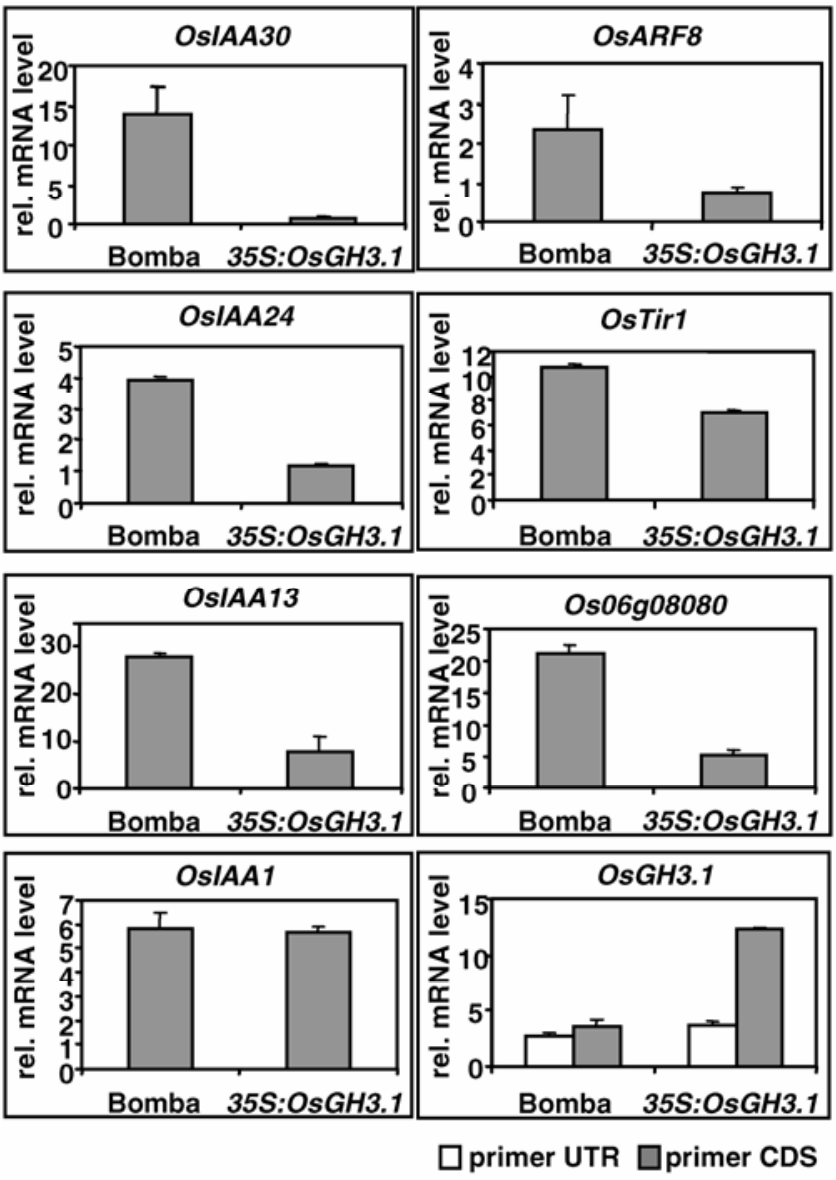

Fig. 3. Expression analysis determined by quantitative reverse-transcriptase polymerase chain reaction analysis of genes related to auxin response in control untransformed plants and 35S:OsGH3.1 plants (line 31). In order to discriminate transgene amounts from endogenous gene levels, OsGH3.1 was amplified with both primers recognizing the untranslated region and primers amplifying a fragment from the coding sequence region. Total RNA was extracted from leaves of 3-week-old plants grown in greenhouse. number of differentially expressed genes was low. Thus, the microarray analysis indicated that $O s G H 3.1$-overexpressing transgenic plants do not particularly induce gene expression related to any specific abiotic stress.

\section{S:OsGH3.1 does not increase tolerance to cold and drought.}

Cold and dehydration tolerance of 35S:OsGH3.1 plants was compared with that of control plants. Untransformed control plants and transgenic plants (lines 8, 13, and 31) were grown hydroponically for 20 days. For cold-tolerance analysis, plants were transferred to and kept at $4^{\circ} \mathrm{C}$ for 48 or $60 \mathrm{~h}$ and then were allowed to recover in nutrient solution at $25^{\circ} \mathrm{C}$ for an additional 9 to 10 days. For drought-tolerance analysis, plants were maintained under dry-air conditions for $12 \mathrm{~h}$ and then were allowed to recover as above. Lines 8, 13, and 31 did not show significant differences in comparison with control plants after 48 or $60 \mathrm{~h}$ of cold treatment (Table 5). Similarly, transgenic lines were no more tolerant to dehydration than control plants (Table 6). The results suggest that $O s G H 3.1$ overexpression has no effect on tolerance to cold and drought stress because reductions in weight among transgenic plants were statistically similar to those observed in controls.

Table 4. Distribution of significant genes related to biotic and abiotic stress based on microarray analyses in transgenic plants overexpressing OsGH3.1

\begin{tabular}{lrrr}
\hline Functional category & UR & DR & Total \\
\hline Biotic stress & 32 & 18 & 50 \\
$\quad$ Response to biotic stimulus & 26 & 10 & 36 \\
Response to pest, pathogen or parasite & 23 & 11 & 34 \\
Defense response & 3 & 2 & 5 \\
Cell death & & & \\
Abiotic stress & 42 & 31 & 73 \\
Response to abiotic stimulus & 3 & 10 & 13 \\
Response to cold & 5 & 8 & 13 \\
Response to salt stress & 9 & 8 & 17 \\
Response to water deprivation & 11 & 1 & 12 \\
Response to wounding & 6 & 0 & 6 \\
Response to carbohydrate stimulus & & & \\
\hline
\end{tabular}

${ }^{\mathrm{z}}$ Genes were grouped in functional categories according to the Gene Ontology classification. UR and DR = up- and downregulated, respectively.

Table 5. Reduction of growth measured as dry weight percentage induced by either 48 or $60 \mathrm{~h}$ of cold treatment $\left(4^{\circ} \mathrm{C}\right)$ in control (untransformed Bomba) and 35S:OsGH3.1 transgenic plants (lines 8, 13, and 31) ${ }^{\mathrm{z}}$

\begin{tabular}{lcc}
\hline Plant & $\mathbf{4 8}$ h cold $(\%)$ & 60 h cold $(\%)$ \\
\hline Bomba & $60.3 \pm 2.7$ & $55.7 \pm 3.5$ \\
Line 8 & $63.6 \pm 7.1$ & $51.4 \pm 6.6$ \\
Line 13 & $70.8 \pm 4.1$ & $62.9 \pm 6.8$ \\
Line 31 & $64.2 \pm 6.1$ & $48.0 \pm 8.6$ \\
\hline
\end{tabular}

${ }^{\mathrm{z}}$ Data are the average \pm standard error of at least 12 plants per line. No statistical differences were found between samples in the same column $(n=50, P<0.05)$

Table 6. Reduction of growth measured as dry weight percentage induced by dehydration in control (untransformed Bomba) and 35S:OsGH3.1 transgenic plants (lines 8,13 , and 31$)^{\mathrm{Z}}$

\begin{tabular}{lc}
\hline Plant & Dehydration stress (\%) \\
\hline Bomba & $44.1 \pm 6.6$ \\
Line 8 & $47.7 \pm 8.2$ \\
Line 13 & $52.2 \pm 2.9$ \\
Line 31 & $46.3 \pm 3.0$ \\
\hline
\end{tabular}

${ }^{\mathrm{z}}$ For dehydration, plants were maintained under dry-air conditions for 12 h. Data are the average \pm standard error of at least 12 plants per line. No statistical differences were found between samples $(n=70, P<0.05)$. 
35S:OsGH3.1 displays resistance to a fungal pathogen.

The finding that several defense-related genes were induced in the transformants led us to examine the resistance of transgenic plants to pathogen infection. In a first bioassay, plants from three transgenic lines overexpressing $O s G H 3.1$ (lines 8, 31, and 36) and untransformed Bomba plants were inoculated with two different European strains (PR14 and IT10) of the fungal pathogen Magnaporthe grisea, the causative agent of rice blast. Results with both races were similar. All three overexpressing lines showed increased defense reaction: the Bomba control had susceptible type lesions with no brown margin whereas all three lines showed intense browning around lesions. In this first assay, the four lines were at different leaf stages when inoculated because transgenic plants showed a significant growth delay. To avoid the influence of leaf stage, which is known to influence resistance to blast in rice, a second assay was performed. In this experiment, overexpressing lines (nos. 13 and 31) were sown 2 days before Bomba and inoculated with one European strain (PR14). Increased defense and minor affected areas were observed again in overexpressing lines that also showed more intense browning around lesions than the untransformed control (Fig. 4). Lesion size was also reduced by 40 to $50 \%$ in $O s G H 3.1$-overexpressing lines compared with Bomba plants (Table 7).

To ascertain the role of auxin in resistance to fungal pathogenesis, 4-week-old 35S:OsGH3.1 plants (line 31) and Bomba untransformed plants were treated with IAA or 2,4-D (a synthetic IAA analogue that is not substrate for IAA-amido synthetases) and, 3 h later, were inoculated with $M$. grisea. Seven days after inoculation, the number of lesion per leaf was scored on 25 leaves (one leaf per plant). Both OsGH3.1-over-

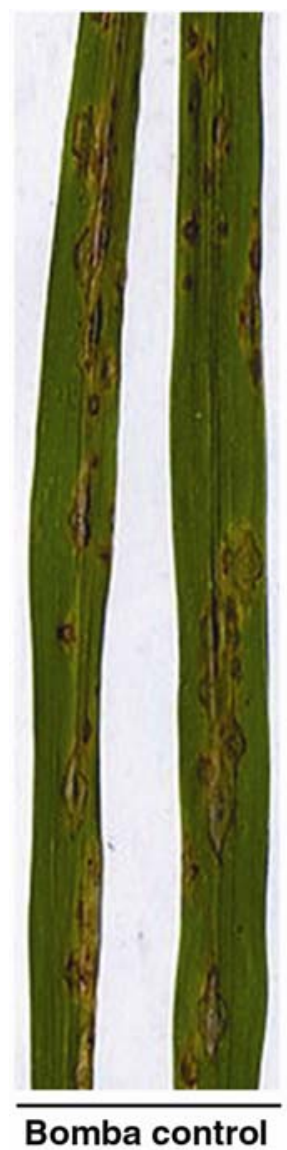

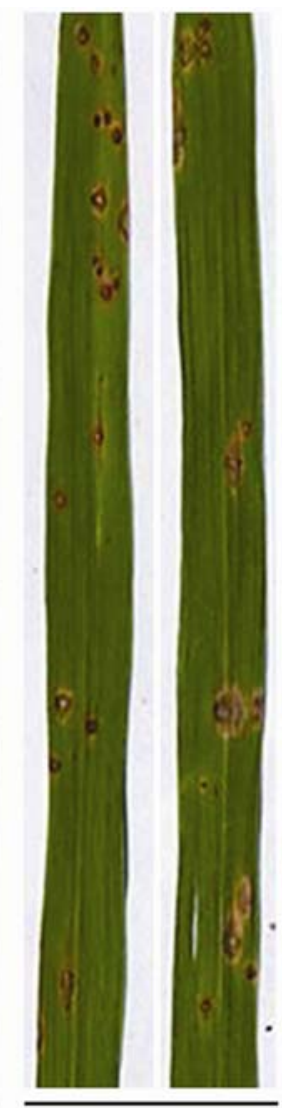

line \#13

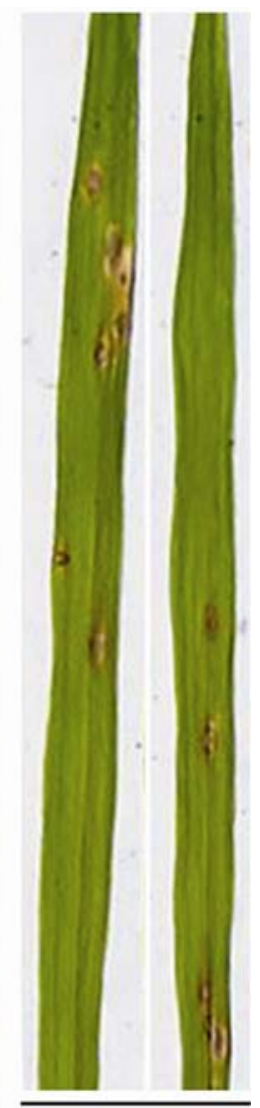

line \#31
Fig. 4. Enhanced resistance to Magnaporthe grisea conferred by overexpression of $O s G H 3.1$. Bomba control and plants from lines 13 and 31 were inoculated with European M. grisea strain (PR14). expressing and untransformed control plants treated with 2,4$\mathrm{D}$ did not show any significant difference in lesion number compared with untreated plants (Table 8). When plants were treated with IAA, similar results were observed. Therefore, no obvious difference in the response to fungal attack could be found upon exogenous application of auxin.

\section{DISCUSSION}

The first relevant finding of this work is that $O s G H 3.1$ is involved in the regulation of free IAA content in rice. Overexpression of $O s G H 3.1$ in transgenic rice plants produced depletion of the free IAA content. This observation is of physiological relevance because it offers an explanation for the dwarf phenotype of the GH3-overexpressing plants, connecting growth with hormonal shortage. The $\mathrm{GH} 3$ gene group was first described as an early auxin-inducible family (Hagen and Guilfoyle 1985). Later, it was proved that a group of the Arabidopsis GH3 genes encoded IAA-amido synthetases that control the endogenous levels of auxin through their capability to conjugate amino acids (Staswick et al. 2005). It has been shown that plants that constitutively express $A R F 8$ had a decrease in free IAA content and, at the same time, higher transcript levels of AtGH3.6 (DFL1), AtGH3.5 (AtGH3a), and AtGH3.17 (Tian et al. 2004). Thus, it was proposed that ARF8 may control the free auxin content through the negative regulation of $G H 3$ gene expression. Therefore, the overexpression of one of these genes may decrease auxin content, disturbing auxin homeostasis. Recently, it has been reported that the overexpression of $O s G H 3.8$ produced a decrease in free IAA level in transgenic plants (Ding et al 2008).

In the work presented here, overexpression of $\mathrm{OsGH3.1}$ produced a reduction in cell elongation, a phenotype consistent with the decrease of free auxin levels. In dwarf Arabidopsis plants with constitutive expression of AtGH3.6 (DFL1), free auxin levels were not significantly decreased, although the level of IAA-Asp conjugate was increased (Staswick et al. 2005). In our work, GC-MS determinations of endogenous IAA showed that the rice transformants certainly contained reduced levels of free and active IAA, suggesting that, in these plants, increased IAA conjugation to amino acids is the main cause of the dwarf phenotype. Transcriptomic analysis of plants overexpressing $O s G H 3.1$ also showed a high proportion of downregulated genes involved in cell growth and cellular

Table 7. Lesion size caused by Magnaporthe grisea infection in control (untransformed Bomba) and 35S:OsGH3.1 transgenic plants (lines 13 and $31)^{\mathrm{z}}$

\begin{tabular}{lc}
\hline Plants & Lesion size $(\mathbf{c m})$ \\
\hline Bomba & $2.9 \pm 1.7 \mathrm{a}$ \\
Line 13 & $1.4 \pm 0.5 \mathrm{~b}$ \\
Line 31 & $1.7 \pm 0.5 \mathrm{~b}$ \\
\hline
\end{tabular}

${ }^{\mathrm{z}}$ Data are the average \pm standard error of 15 plants per line. Identical letters show no statistical differences $(n=45, P<0.05)$.

Table 8. Number of lesions per leaf caused by Magnaporthe grisea infection in control (untransformed Bomba) and 35S:OsGH3.1 transgenic plants (line 31$)^{\mathrm{Z}}$

\begin{tabular}{lccc}
\hline Plants & Untreated & $\begin{array}{c}\mathbf{1 0 0} \boldsymbol{\mu M} \text { indole-3- } \\
\text { acetic acid }\end{array}$ & $\begin{array}{c}\mathbf{1 0 0} \boldsymbol{\mu M} \\
\mathbf{2 , 4 - D}\end{array}$ \\
\hline Bomba & $2.9 \pm 0.7 \mathrm{ab}$ & $1.5 \pm 0.4 \mathrm{~b}$ & $4.0 \pm 0.8 \mathrm{a}$ \\
Line \#31 & $1.0 \pm 0.4 \mathrm{a}$ & $1.1 \pm 0.2 \mathrm{a}$ & $1.6 \pm 0.5 \mathrm{a}$ \\
\hline
\end{tabular}

${ }^{\mathrm{z}}$ Data are the average \pm standard error of at least 20 plants per line and treatment. Identical letters in the same row show no statistical differences $(n=63, P<0.05)$. 
morphogenesis. Most of these latter genes were related to cell wall biosynthesis (glycosyl hydrolases 1,4- $\beta$-glucanases) or cell wall loosening (several expansins), indicating that both processes were inhibited. This is an expected observation that correlated with the dwarf phenotype exhibited by these transformants.

The decrease in free IAA content in transgenic plants was accompanied by significant repression of transcript levels of several $A u x / I A A$ genes and $A R F 8$ and also by slight downregulation of TIR1. The Aux/IAA family is a group of primary auxin-responsive genes that mediates responses to auxin, inhibiting transcription of auxin-inducible genes by forming inactive heterodimers with the auxin response factor (ARF) family of DNA-binding proteins (Tiwari et al. 2003). As mentioned above, ARF bind to auxin-responsive elements of primary auxin-response genes and activate (or repress) transcription. Aux/IAA proteins are short-lived nuclear proteins whose degradation is proteasome dependent and mediated by the Fbox protein TIR1, which is part of the ubiquitin $\mathrm{SCF}^{\mathrm{TIR} 1}$ complex and has the capacity to bind auxin (Gray et al. 2001; Kepinski and Leyser 2005). Later on, as an alternative to TIR1, it was proposed that posttranscriptional cleavage of ARF mRNA by microRNA also modulates auxin signaling (Yang et al. 2006). Thus, Aux/IAA proteins function as ARF inhibitors, decreasing auxin signaling (Nakamura et al. 2006). In the presence of an inducible level of auxin, ARF homodimerize while Aux/IAA proteins are proteolyzed via the ubiquitin pathway. As a consequence of the depletion of auxin signaling, transcript levels of both TIR1 and ARF8 are diminished.

It is currently accepted that there are complex interactions between different hormones and environmental stimuli. The relationship between auxin and ethylene, gibberellic acid, and brassinosteroids has recently been documented (Woodward and Bartel 2005), for instance. Furthermore, the pleiotropic defects of mutants affected in components of signaling pathways strongly indicate intercommunication between several hormone response pathways. Examples of these interactions are mutations in $A u x / I A A$ and $A R F$ genes that have impaired light responses, RUB E2 enzyme-defective mutants that are auxin and methyl jasmonate resistant and also overproduce ethylene, or mutations in the auxin transport facilitator TIR3 that have altered response to auxin, light, ethylene, cytokinin, and giberellin (Kanyuka et al. 2003; Lasen and Cancel 2004; Tian et al. 2004; Woodward and Bartel 2005). Other important examples are the DELLA proteins that have been reported to be integrators of plant responses to independent hormonal and environmental signals (Achard et al. 2006) and OsNAC6 that functions as a transcriptional activator induced by different abiotic factors as well as by infection with the blast fungus (Nakashima et al. 2007). Transcriptomic analysis of rice plants overexpressing $O s G H 3.1$ showed a considerable number of differentially expressed genes related to abiotic stimulus, many of them also associated with two or more stress inductors. Thus, many upregulated genes related to the response of wounding and cold were also included in the group of genes involved in the response to biotic agents. This observation indicates the occurrence of cross-talk between different signaling pathways implicated in the biotic and abiotic response of the plant to the environment. Transgenic plants overexpressing OsGH3.1, however, did not show differential response to both cold and desiccation treatments, indicating that factors other than auxin content are involved in the tolerance to these specific stresses.

Transcriptomic analyses also revealed a considerable number of induced defense response genes, another pivotal observation of this work because it links IAA content depletion with enhanced defense response. It is well established that several plant hormones such as SA, JA, and ethylene are main regula- tors of the signal pathways that activate defense responses in several plant species (Anderson et al. 2004; McConn et al. 1997; Ryals et al. 1996). However, the role of SA in rice remains elusive because rice contains high basal levels of SA compared with Arabidopsis or tobacco and appears to be insensitive to exogenous SA treatment (Yang et al. 2004). In rice, SA does not appear to act as an effective signal molecule to activate disease resistance although it has been suggested that it could play an antioxidative role to protect rice plants from oxidative stress (Yang et al 2004). Recent investigations have pointed out that auxin homeostasis might also be one of the components participating in the regulation of plant defense. Recently, it has been reported that virulence factors from pathogenic bacteria $P$. syringae modulate host auxin physiology to promote disease in Arabidopsis (Chen et al. 2007; Navarro et al. 2006). More recently, a role in bacterial resistance was proposed for OsGH3.8, an IAA-amino synthetase, that would act as an activator of disease resistance by inducing a transient and cell-specific suppression of auxin signaling (Ding et al. 2008). In the case of viral agents, other relevant examples can be found in the literature relating auxin depletion and pathogen resistance (Mayda et al. 2000; Padmanabhan et al. 2005, 2006). On the other hand, the transcription factor OsWRKY31 has been proposed as a common component in the signal transduction pathways of auxin response and defense in rice (Zhang et al. 2008). These previous works strongly suggested the occurrence of an interactive cross-talk system between auxin homeostasis and pathogen elicitation in both monocot and dicots.

Plant defense is a costly event and plants have evolved to minimize this effort by integrating growth responses to environmental and pathogen signals. In this context, coevolution of pathogen and plants led to an interplay in which the pathogen tries to manipulate its host by unbalancing the hormonal homeostasis, increasing auxin signaling to promote disease and, meanwhile, the plants respond by downregulating auxin signaling that could act as an immune response by inhibiting cell wall loosening and activating defense genes. In this regard, the ability of several members of the OsGH3 family to conjugate free IAA may contribute to plant defense. In the work reported here, OsGH3.1 overexpression produced a depletion of auxin signaling leading to the activation of a plethora of defense genes, including those related to phenylpropanoid and terpenoid metabolism. The transcriptomic profile also revealed downregulation of genes implicated in loosening and biosynthesis of cell walls, two processes that may debilitate the function of these natural barriers against fungal and bacterial attack. Moreover, the results presented in this work showed that exogenous auxins cannot restore or increase susceptibility in tolerant transgenic or wild-type plants, indicating that the auxin content per se may not be ultimately responsible for the tolerance observed in the transgenic plants. Rather, the results suggest that the cause of the loss of susceptibility is likely associated with one or several initial effects of auxin deficiency. The inhibition of cell wall loosening, linked here to the dwarf phenotype, which was not restored by exogenous auxins, for instance, might contribute to the defense of the plant against pathogens.

In conclusion, data reported in this article extend and complement the concept developed in previous work that suggests that auxin homeostasis participates in the regulation of plantinduced immune response by activating defense genes. These OsGH3.1-overexpressing lines offered an opportunity to further investigate the role of auxin in triggering defense against a fungal pathogen (M. grisea) in a monocot species (rice). It is shown that $O s G H 3.1$ overexpression in rice reduces free auxin content and activates defense response genes. Resistant assays 
with the fungal pathogen $M$. grisea demonstrated that these auxin-deficient plants also showed enhanced fungal resistance. This observation provides correlative evidence among auxin homeostasis and the mechanism of defense response in rice.

\section{MATERIALS AND METHODS}

Plant material and growth conditions.

Rice (Oryza sativa subsp. japonica) cv. Bomba was used in all studies. Plants were grown in summer in the greenhouse under natural daylight at $25^{\circ} \mathrm{C}$. For microarray expression analysis, the aerial parts of 6-week-old plants were frozen in liquid nitrogen and stored at $-80^{\circ} \mathrm{C}$ until RNA isolation. For physiological experiments, T2 seed were germinated during 2 days in water and selected in agar dishes in the presence of hygromicin $(40 \mathrm{mg} / \mathrm{liter})$, then transferred to nutrient solution (Yoshida et al. 1976) and grown in growth chambers at $25^{\circ} \mathrm{C}$ during a 12-h photoperiod with relative humidity of $75 \%$.

The height of adult plants was determined by measuring the length of the main tiller of each transgenic plant from the crown of the roots to the flag leaf.

\section{Auxin extraction, purification, and quantitation.}

Indolacetic extraction from leaf samples was performed with $80 \%$ methanol. After purification with SS-Sax and $\mathrm{C}_{18}$ Sep-Pak cartridges, samples were dried to vacuum and further fractionation performed with reverse-phase high-performance liquid chromatography. Fractions containing IAA were evaporated to dryness, then methylated with ethereal diazomethane and tri-methyl-silylated at room temperature for at least $1 \mathrm{~h}$ with 5 to $10 \mu \mathrm{l}$ of bis-trimethylsilyl-trifluoroacetamide.

Analysis of IAA was performed by GC-MS using a gas chromatograph (Star $3400 \mathrm{CX}$; Varian, Sunnyvale, CA, U.S.A.) coupled to an ion trap mass spectrometer (Saturn 3; Varian) in the splitless mode into a fused silica capillary column. The $\mathrm{He}$ inlet pressure was $60 \mathrm{kPa}$ and the injector, interface and manifold temperatures were 250,250 , and $200^{\circ} \mathrm{C}$, respectively. For quantitation purposes, $\left[{ }^{2} \mathrm{H}_{2}\right]$-IAA was added to the extracts as an internal standard. Abundance of IAA was monitored through calculation of the area ratio of ions at $\mathrm{m} / \mathrm{z} 202$ and 204 of IAA and $\left[{ }^{2} \mathrm{H}_{2}\right]$-IAA, respectively, in the chromatographic profiles. In preliminary analyses, various amounts of internal standards were added to the extracts in consecutive extractions and the produced IAA amounts were initially estimated. This strategy was repeated until the amount of internal standards and the amount of endogenous IAA was similar. All quantitations were independently repeated on at least three different samples from leaves of T1 adult plants or from leaves of six different plants of each $\mathrm{T} 2$ line.

\section{Rice transformation.}

OsGH3.1 was amplified by PCR from genomic DNA from cv. Bomba using the primers 5'-CCCATGGCGGAAGCAC CGACCGCC and 5'-GACTAGTCGACCAATGACCATATGC ATG. The PCR product was digested with NcoI and SpeI and cloned under the $35 \mathrm{~S}$ promoter in pCAMBIA1305.1 (Cambia, Australia) to generate p35S:OsGH3.1. The resultant plasmid was transformed into Agrobacterium tumefaciens EHA105 by electroporation, and used for the transformation of Bomba rice (subsp. japonica). Rice transformation was performed according to Hiei and associates (1994).

\section{RNA extraction, RNA amplification, and} microarray hybridization.

Total RNA was isolated with Trizol (Invitrogen, Carlsbad, CA, U.S.A.) and further purified with the RNeasy midi kit (Qiagen, Valencia, CA, U.S.A.). RNA samples were treated with RNase free DNase I (Invitrogen). Aliquots of RNA were kept to perform semiquantitative and Q-RT-PCR analysis. The RNA amplification and labeling were performed with the AminoAllyl MessageAmp II aRNA Amplification kit (Ambion, Austin, TX, U.S.A.) according to the manufacturer's instructions. Total RNA of each sample $(1 \mu \mathrm{g})$ was used for the amplification. The final concentration of obtained amplified RNA (aRNA) was determined with a NanoDrop ND1000 Spectrophotometer. For the dye-coupling reaction, $10 \mu \mathrm{g}$ of aRNA of each sample were used following the "Dye Coupling and Labeled aRNA Cleanup" protocol (Ambion). aRNA of each sample $(2 \mu \mathrm{g})$ was fragmented with the Ambion's RNA fragmentation reagents (Ambion).

The Rice NSF oligonucleotide microarray $20 \mathrm{~K}$ (50 to 70 mers), containing 20,230 TIGR version 2.0 gene models, was used for gene expression detection. Additional information about this array is available at the NSF Rice Oligonucleotide Array Project web page. The probes for the hybridizations consisted of aRNA of three distinct individuals from the transgenic line 31 and three other distinct individuals from wild-type plants. Each sample was labeled twice, once with $\mathrm{Cy} 3$ and the other time with Cy5, and, therefore, was directly compared against two samples of the other genotype (Kerr and Churchill 2001). Hybridization overnight at $42^{\circ} \mathrm{C}$, washing, and scanning (ScanArray Gx; Perkin Elmer, Wellesley, MA, U.S.A.) was carried out exactly as described at the NSF Rice Oligonucleotide Array Project website. All microarray data described in this study were deposited into the ArrayExpress database (accession number A-MEXP-1386 and E-MEXP-1773).

\section{Microarray data analysis.}

Image analysis was performed using GenePix 4.1 Pro software (Axon Instruments, Foster City, CA, U.S.A.). Quality control, normalization, and determination of differentially expressed genes were performed in $\mathrm{R}$ using the Limma package (Smyth 2005) of Bioconductor. Background intensity in each spot was subtracted from foreground intensity using the "normexp" method and the normalization step was carried out using the "vsn" method (Smyth and Speed 2003). Because each individual was represented on two different arrays, the hybridizations were not independent. This circumstance was taken into account to obtain the list of differential expressed genes, following the procedures previously described in Smyth (2004). The list was filtered to select candidate genes with $P$ value $<$ 0.001 and a ratio of intensities between transgenic and wildtype plants of $-1<\mathrm{M}>1\left(\mathrm{M}=\log _{2}\right.$ [35S:OsGH3.1/Bomba)]). For determining the false discovery rate (FDR), $P$ values were adjusted by multiple testing using Benjamini and Hochberg's method (Benjamini and Hochberg 1995). An adjusted $P$ value greater than $0.001(\mathrm{FDR}=0.1 \%)$ was obtained for all genes. Functional category classifications were grouped using the Blast2GO software (Conesa et al. 2005).

\section{Real-time Q-RT-PCR experiments.}

Aliquots of RNA prepared for the microarray analysis were subjected to real-time Q-RT-PCR. The RNA concentration was determined by a fluorometric assay with the kit Quant-iT RiboGreen RNA assay (catalog number R11490; Molecular Probes Inc., Eugene, OR, U.S.A.) following the manufacturer's instructions. The one-step Q-RT-PCR assays were performed on a LightCycler 2.0 (Roche Applied Science, GE, Mannheim Germany) using the LightCycler Fast Start DNA MasterPlus Sybr Green I kit (cat. no. 03515885001; Roche Applied Science). To each reaction was added 2.5 units of MultiScribe Reverse Transcriptase (cat. no. N8080018; Applied Biosystems, Foster City, CA, U.S.A.), 1 unit of RNase Inhibitor (cat. no. N8080119; Applied Biosystems), $2 \mu \mathrm{l}$ of LC Fast Start DNA 
MasterPlus Sybr Green I, $0.5 \mu \mathrm{l}$ of each primer $(0.25 \mu \mathrm{M}$ final concentration), and $100 \mathrm{ng}$ of total RNA in a total volume of $10 \mu$. The RT-PCR procedure consisted in an incubation at $48^{\circ} \mathrm{C}$ for $30 \mathrm{~min}$, followed of 45 cycles at $95^{\circ} \mathrm{C} 2 \mathrm{~s}, 60^{\circ} \mathrm{C} 8 \mathrm{~s}$, and $72^{\circ} \mathrm{C} 8 \mathrm{~s}$. The identities of the amplicons and the specificity of the reaction were verified by melting curve analysis and by sequencing the reaction product, respectively. To transform fluorescent intensity measurements into relative mRNA levels, a 10-fold dilution series of a RNA sample was used as standard curve. Relative mRNA levels were then normalized to total RNA amounts as previously described (Cercós et al. 2006). The values presented are the mean of the two biological replicates, each with three technical replicates. The error bars indicate the standard deviation from the mean.

\section{Histological analysis.}

Sheath segments from second leaves of adult plants immersed in $80 \%$ (wt/wt) lactic acid were autoclaved for $20 \mathrm{~min}$ at $121{ }^{\circ} \mathrm{C}$. Samples were mounted in fresh $80 \%$ (wt/wt) lactic acid and viewed with a Leica ASLMD microscope. Images were processed using Adobe Photoshop 5.5 (Adobe Systems, Mountain View, CA, U.S.A.). Leaves from three different T2 plants were examined and 20 cells from each one were measured.

\section{Cold and drought treatments and evaluation of resistance to blast disease.}

Seedlings of both control and transgenic plants were grown hydroponically in nutrient solution for 20 days. Then, for cold treatment, plants were transferred to and kept at $4{ }^{\circ} \mathrm{C}$ for either 48 or 60 h. For dehydration treatment, nutrient solution was removed; roots were dried onto filter paper and then kept dried for $12 \mathrm{~h}$. After each treatment, plants were transferred to normal growth condition for 9 days to allow plant recovery. Then, plants were collected and dried in a $60^{\circ} \mathrm{C}$ oven for 2 days, and leaves and stems were weighted. Each treatment was independently performed twice and 12 seedlings per treatment and line were analyzed.

Plants were grown in the greenhouse as previously described (Roumen et al. 1997). Three-week-old plants were inoculated with a $M$. grisea spore suspension calibrated at 50,000 spores/ml. The two M. grisea strains (IT10 and PR14) used to evaluate resistance correspond to two different European races of the blast pathogen (Roumen et al. 1997). Symptoms were observed 1 week post inoculation, leaves were collected for storage, and the size of 15 different lesions per plant was measured. For analysis of the effect of IAA on disease, 3week-old plants were sprayed with $100 \mu \mathrm{M}$ IAA, $100 \mu \mathrm{M} 2,4-$ $\mathrm{D}$, or water $3 \mathrm{~h}$ before they were inoculated with $M$. grisea. Lesion number was scored 7 days post inoculation.

Parameters were statistically tested by analyses of variance and comparisons of means were determined through the least significant difference method. Statistical analyses were performed with StatGraphics Plus for Windows (version 2.1; Statistical Graphics, Englewood Cliffs, NJ, U.S.A.).

\section{ACKNOWLEDGMENTS}

This work has been supported by Ministerio de Educación y Ciencia grant GEN2006-27764-C4-1-E/VEG. C. Domingo was supported by the Instituto Nacional de Investigación y Tecnología Agraria y Alimentaria. We thank S. Vancoppenolle and C. Tertois for technical assistance.

\section{LITERATURE CITED}

Achard, P., Cheng, H., De Grauwe, L., Decat, J., Schoutteten, H., Moritz, T., Van Der Straeten, D., Peng, J., and Harberd, N. P. 2006. Integration of plant responses to environmentally activated phytohormonal signals. Science 311:91-94.
Anderson, J. P., Badruzsaufari, E., Schenk, P. M., Manners, J. M., Desmond, O. J., Ehlert, C., Maclean, D. J., Ebert, P. R., and Kazan, K. 2004. Antagonistic interaction between abscisic acid and jasmonate-ethylene signaling pathways modulates defense gene expression and disease resistance in Arabidopsis. Plant Cell 16:3460-3479.

Benjamini, Y., and Hochberg, Y. 1995. Controlling the false discovery rate: A practical and powerful approach to multiple testing. J. Roy. Stat. Soc. B 57:289-300.

Cercós, M., Soler, G., Iglesias, D. J., Gadea, J., Forment, J., and Talon, M. 2006. Global analysis of gene expression during development and ripening of citrus fruit flesh. A proposed mechanism for citric acid utilization. Plant Mol. Biol. 62:513-527.

Chen, Z., Agnew, J. L., Cohen, J. D., He, P., Shan, L., Sheen, J., and Kunkel, B. N. 2007. Pseudomonas syringae type III effector AvrRpt2 alters Arabidopsis thaliana auxin physiology. Proc. Natl. Acad. Sci. U.S.A. 104:20131-20136.

Conesa, A., Gotees, S., García-Gómez, J. M., Terol, J., Talon, M., and Robles, M. 2005. Blast2GO: A universal annotation and visualization tool in functional genomics research. Application note. Bioinformatics 21:3674-3676.

Ding, X., Cao, Y., Huang, L., Zhao, J., Xu, C., Li, X., and Wang, S. 2008. Activation of the indole-3-acetic acid-amido synthetase GH3-8 suppresses expansin expression and promotes salicylate- and jasmonateindependent basal immunity in rice. Plant Cell 20:228-240.

Fu, X., and Harberd, N. P. 2003. Auxin promotes Arabidopsis root growth by modulating gibberellin response. Nature 421:740-743.

Glickmann, E., Gardan, L., Jacquet, S., Hussain, S., Elasri, M., Petit, A., and Dessaux, Y. 1998. Auxin production is a common feature of most pathovars of Pseudomonas syringae. Mol. Plant-Microbe Interact. 11:156-162.

Gray, W. M., Kepinski, S., Rouse, D., Leyser, O., and Estelle, M. 2001. Auxin regulates SCF(TIR1)-dependent degradation of AUX/IAA proteins. Nature 414:271-276.

Gutierrez-Mañero, F. J., Ramos-Solano, B., Probanza, A., Mehouachi, J., Tadeo, F. R., and Talon, M. 2001. The plant-growth-promoting rhizobacteria Bacillus pumilus and Bacillus licheniformis produce high amounts of physiologically active gibberellins. Physiol. Plant. 111:206211.

Hagen, G., and Guilfoyle, T. J. 2002. Auxin-responsive gene expression: Genes, promoters and regulatory factors. Plant Mol. Biol. 49:373-385.

Halliday, K. 2004. Plant hormones: The interplay of brassinosteroids and auxin. Curr. Biol. 14:R1008-R1010.

Hiei, Y., Ohta, S., Komari, T., and Kumashiro, T. 1994. Efficient transformation of rice (Oryza sativa L.) mediated by Agrobacterium and sequence analysis of the boundaries of the T-DNA. Plant J. 6:271-282.

Idris, E., Iglesias, D., Talon M., and Borriss, R. 2007. Tryptophan-dependent production of indole-3-acetic acid (IAA) affects level of plant growth promotion by Bacillus amyloliquefaciens FZB42. Mol. PlantMicrobe Interact. 20:619-626.

Jagadeeswaran, G., Raina, S., Acharya, B. R., Maqbool, S. B., Mosher, S. L., Appel, H. M., Schultz, J. C., Klessig, D. F., and Raina, R. 2007. Arabidopsis GH3-LIKE DEFENSE GENE 1 is required for accumulation of salicylic acid, activation of defense responses and resistance to Pseudomonas syringae. Plant J. 51:234-246.

Jain, M., Kaur, N., Tyagi, A., and Khurana, J. 2006a The auxin-responsive $\mathrm{GH} 3$ gene family in rice Oryza sativa. Funct. Integr. Genomics 6:36-46.

Jain, M., Kaur, N., Garg, R., Thakur, J. K., Tyagi, A. K., and Khurana, J. P. $2006 \mathrm{~b}$ Structure and expression analysis of early auxin-responsive Aux/IAA gene family in rice (Oryza sativa) Funct. Integr. Genomics 6:47-59.

Kanyuka, K., Praekelt, U., Franklin, K. A., Billingham, O. E., Hooley, R., Whitelam, G. C., and Halliday, K. J. 2003. Mutations in the huge Arabidopsis gene $B I G$ affect a range of hormone and light responses. Plant J. 35:57-70.

Kepinski, S., and Leyser, O. 2005. The Arabidopsis F-box protein TIR1 is an auxin receptor. Nature 435:446-451.

Kerr, M. K., and Churchill, G. A. 2001. Statistical design and the analysis of gene expression microarray data Genet. Res. 77:123-128.

Larsen, P. B., and Cancel, J. D. 2004. A recessive mutation in the RUB1conjugating enzyme, $R C E 1$, reveals a requirement for RUB modification for control of ethylene biosynthesis and proper induction of basic chitinase and PDF1.2 in Arabidopsis. Plant J. 38:626-638.

Leyser, O. 2006. Molecular genetics of auxin signaling. Annu. Rev. Plant Biol. 53:377-398.

Mayda, E., Marqués, M. C., Conejero, V., and Vera, P. 2000. Expresión of a pathogen-induced gene can be mimicked by auxin insensitivity. Mol. Plant-Microbe Interact. 13:23-31.

McConn, M., Creelman, R. A., Bell, E., Mullet, J. E., and Browse, J. 1997. Jasmonate is essential for insect defense in Arabidopsis. Proc. Natl. Acad. Sci. U.S.A. 94:5473-5477. 
Nakamura, A., Umemura, I., Gomi, K., Hasegawa, G., Kitano, H., Sazuka, T., and Matsuoka, M. 2006. Production and characterization of auxininsensitive rice by overexpression of a mutagenized rice IAA protein. Plant J. 46:297-306.

Nakashima, K., Tran, L.-S. P., Van Nguyen, D., Fujita, M., Maruyama, K. Todaka, D., Ito, Y., Hayashi, N., Shinozaki, K., and YamaguchiShinozaki, K. 2007. Functional analysis of a NAC-type transcription factor OsNAC6 involved in abiotic and biotic stress-responsive gene expression in rice. Plant J. 51:617-630.

Nakazawa, M., Yabe, N., Ichikawa, T., Yamamoto, Y. Y., Yoshizumi, T., Hasunuma, K., and Matsui, M. 2001. DFL1, an auxin-responsive GH3 gene homologue, negatively regulates shoot cell elongation and lateral root formation, and positively regulates the light response of hypocotyl length. Plant J. 25:213-221.

Navarro, L., Dunoyer, P., Jay, F., Arnold, B., Dharmasiri, N., Estelle, M., Voinnet, O., and Jones J. D. G. 2006. Plant miRNA contributes to antibacterial resistance by repressing auxin signaling. Science 312:436-439.

Nobuta, K., Okrent, R. A., Stoutemyer, M., Rodibaugh, N., Kempema, L. Wildermuth, M. C., and Innes, R. W. 2007. The GH3 acyl adenylase family member PBS3 regulates salicylic acid-dependent defense responses in Arabidopsis. Plant Physiol. 144:1144-1156.

Padmanabhan, M. S., Goregaoker, S. P., Golem, S., Shiferaw, H., and Culver, J. N. 2005. Interaction of the tobacco mosaic virus replicase protein with the Aux/IAA protein PAP1/IAA26 is associated with disease development. J. Virol. 79:2549-2558.

Padmanabhan, M. S., Shiferaw, H., and Culver, J. N. 2006. The tobacco mosaic virus replicase protein disrupts the localization and function of interacting Aux/IAA proteins. Mol. Plant-Microbe Interact. 19:864-873.

Roumen, E., Levy, M., and Notteghem, J. L. 1997. Characterisation of the European pathogen population of Magnaporthe grisea by DNA fingerprinting and pathotype analysis. Eur. J. Plant Pathol. 103:363-371.

Ryals, J. A., Neuenschwander, U. H., Willits, M. G., Molina, A., Steiner, H.-Y., and Hunt, M. D. 1996 Systemic acquired resistance. Plant Cell 8:1809-1819.

Silverman, P., Seskar, M., Kanter, D., Schweizer, P., Metraux, J.-P., and Raskin, I. 1995. Salicylic acid in rice: Biosynthesis, conjugation, and possible role. Plant Physiol. 108:633-639.

Smyth, G. K. 2004. Linear models and empirical Bayes methods for assessing differential expression in microarray experiments. Stat. Appl. Genet. Mol. Biol. 3:Article 3.

Smyth, G. K. 2005. Limma: Linear models for microarray data. Pages 397-420 in: Bioinformatics and Computational Biology Solutions using R and Bioconductor. R. Gentleman, V. Carey, S. Duboit, R. Irizarry, W. Huber, eds. Springer, New York.

Smyth, G. K., and Speed, T. P. 2003. Normalization of cDNA microarray data. Methods 31:265-273.

Staswick, P. E., and Tiryaki, I. 2004. The oxylipin signal jasmonic acid is activated by an enzyme that conjugates it to isoleucine in Arabidopsis. Plant Cell 16:2117-2127.

Staswick, P. E., Serban, B., Rowe, M., Tiryaki, I., Maldonado, M. T., Maldonado, M. C., and Suza, W. 2005. Characterization of an Arabi- dopsis enzyme family that conjugates amino acids to indole-3-acetic acid. Plant Cell 17:616-627.

Takase, T., Nakazawa, M., Ishikawa, A., Kawashima, M., Ichikawa, T., Takahashi, N., Shimada, H., Manabe, K., and Matsui, M. 2004. ydk1-D, an auxin-responsive $G H 3$ mutant that is involved in hypocotyl and root elongation. Plant J. 37:471-483.

Teale, W. D., Paponov, I. A., and Palme, K. 2006. Auxin in action: Signaling, transport and the control of plant growth and development. Nat. Rev. Mol. Cell Biol. 7:847-859.

Terol, J., Domingo, C., and Talon, M. 2006. The GH3 family in plants: Genome wide analysis in rice and evolutionary history based on EST analysis. Gene 371:279-290.

Tian, C. E., Muto, H., Higuchi, K., Matamura, T., Tatematsu, K., Koshiba, T., and Yamamoto, K. T. 2004. Disruption and overexpression of auxin response factor 8 gene of Arabidopsis affect hypocotyl elongation and root growth habit, indicating its possible involvement in auxin homeostasis in light condition. Plant J. 40:333-343.

Tiwari, S. B., Hagen, G., and Guilfoyle, T. 2003. The roles of auxin response factor domains in auxin-responsive transcription. Plant Cell 15:533-543.

Wang, D., Pajerowska-Mukhtar, K., Culler, A. H., and Dong, X. 2007. Salicylic acid inhibits pathogen growth in plants through repression of the auxin signaling pathway. Curr. Biol. 17:1784-1790.

Woodward, A. W., and Bartel, B. 2005. Auxin: Regulation, action and interaction. Ann. Bot. 95:707-735.

Yang, J. H., Han, S. J., Ion, E. K., and Lee, W. S. 2006. Evidence of an auxin signal pathway, microRNA167-ARF8-GH3, and its response to exogenous auxin in cultured rice cells. Nucleic Acids Res. 34:1892 1899

Yang, Y., Qi, M., and Mei, C. 2004. Endogenous salicylic acid protects rice plants from oxidative damage caused by aging as well as biotic and abiotic stress. Plant J. 40:909-919.

Yoshida, S., Forno, D. A., Cock, J. H., and Gomez, K. A. 1976. Pages 6166 in: Laboratory Manual for Physiological Studies of Rice. International Rice Research Institute, Manila, The Philippines.

Zhang, Z., Li., Q, Li, Z., Staswick, P., Wang, M., Zhu, Y., and He, Z. 2007. Dual regulation role of GH3.5 in salicylic acid and auxin signaling during Arabidopsis-Pseudomonas syringae interaction. Plant Physiol. 145:450-464

Zhang, J., Peng, Y., and Guo, Z. 2008. Constitutive expression of pathogen-inducible OsWRKY31 enhances disease resistance and affects roo growth and auxin response in transgenic rice plants. Cell Res. 18:508521.

\section{AUTHOR-RECOMMENDED INTERNET RESOURCES}

NSF Rice Oligonucleotide Array Project website: www.ricearray.org The TIGR Arabidopsis thaliana Genome Project database: www.tigr.org/tdb/e2k1/ath1/ath1.shtml

Bioconductor website: www.bioconductor.org 